\title{
EFFECTS OF LANE DEPARTURE WARNING ON DROWSY DRIVERS' PERFORMANCE AND STATE IN A SIMULATOR
}

\author{
Maria Rimini-Doering, Tobias Altmueller, \\ Ulrich Ladstaetter, Markus Rossmeier \\ Corporate Research, Robert Bosch GmbH \\ Stuttgart, Germany \\ E-mail: maria.rimini-doering@de.bosch.com
}

\begin{abstract}
Summary: Driver drowsiness is a major cause of severe accidents, many of which involve a single vehicle lane departure. The objective of the experiment described in this paper is to determine the relationships between drowsiness, lane departure events (LDE) and effects of a warning system. While in case of driver distraction the impact of such a warning system can be tested in real traffic, for reasons of safety (and reproducibility), a laboratory-based driving simulator is being used in this project. The experiments were conducted with a cohort of 63 healthy male subjects aged 22 to 27 driving for about $2.5 \mathrm{hrs}$ in a stimuli-deprived scenario with a six-fold repetition under carefully controlled conditions. Several hundreds micro-sleep episodes were identified in the 53 successful trials by electrooculogram and video signal and confirmed by behavioral analysis; more than 800 lane departure warnings (LDW) occurred in the assisted sub-cohort of 17 drivers. A combined analysis of the LDE with and without LDW shows significant reduction in number, time, departure length and out-of-lane area for the assisted subjects. The timing and design of the warning could furthermore prevent almost $85 \%$ of the lane departure events caused by sleepiness.
\end{abstract}

\section{INTRODUCTION}

Addressing the problem of traffic casualties (currently 50,000/year), the European Union has set a very ambitious goal of cutting them by $50 \%$ before year 2010 in the enlarged boundaries of the Union (European Commission, 2001). Driver drowsiness together with distraction and workload in complex vehicle and traffic environments has long been identified as one of the primary single causes of accidents (Langwieder, 1994). Research in the last years has studied the drowsiness phenomenon under different perspectives, on the one hand trying to understand the process and indicators of such a state (Rimini-Doering, 2001) and on the other developing assistance systems that alarm the driver, correct eventual errors or mitigate their unavoidable consequences. Lane Departure Warning Systems (LDWS) alert the driver once he/she is approaching the border of the lane under well-defined circumstances (e.g., Motoyama, 2000). While a real traffic environment offers a variety of conditions for testing the consequences of distraction and workload on lane keeping performance, for obvious reasons of safety and reproducibility, the test of a LDWS in case of drowsiness has to be performed in a driving simulator.

\section{Objective}

The primary objective of this project is to induce a large number of drowsiness events (microsleep episodes) under carefully controlled conditions for mainly two reasons: study the onset and the process of becoming drowsy by analyzing both physiological parameters and driving 
performance and at the same time compare the behavior of two different sub-cohorts, with and without the assistance of an LDWS. In this paper we will concentrate on the second aspect of the results: quantify the effects of the LDWS on performance and state of the driver.

\section{EXPERIMENT EQUIPMENT}

\section{Driving Simulator}

A fixed-platform driving simulator is used for implementing the driving task. The driver sits in front of a $135^{\circ}$ screen in an equipped front half of a car mock-up, with force feedback steering wheel, acceleration and brake pedals for automatic mode. The simulator software is based on Stisim 500W from Systems Technology, Inc. (Allen, 1998), running on a local network of four Pentium IV computers with a clock speed between 2.0 and $2.4 \mathrm{GHz}$. A 17 degree of freedom model computes the vehicle dynamics to which the animated scenes respond. Dedicated sound cards generate car noise as a function of vehicle and motor speed, as well as the stereo warning signal for the LDWS. In our experiments, up to 40 driving and dynamic parameters such as steering wheel angle, pedal inputs, speed, lane position and heading angle are logged with a sampling rate of $100 \mathrm{~Hz}$. The simulator receives a Gray encoded reference-time axis to ensure accurate synchronization (in the order of ppm) with the external physiological and video measurement equipment (Altmueller, 2003, [3]).

\section{Sensors}

Physiological sensors. Two MP150 recording systems by Biopac Systems, Inc., records physiological parameters, such as electroencephalogram (EEG), electrocardiogram (ECG), electrooculogram (EOG), skin conductivity (EDA) and temperature, with dedicated amplifiers for each channel. The sampling rate of $100 \mathrm{~Hz}$ matches the simulator data rate, while time reference information is similarly fed into the system for synchronization.

Eye-tracking sensors. Eye movements and closures as well as head movements are tracked by faceLAB, a stereo camera-based image processing system from Seeing Machines, Inc. Several derived measures such as PERCLOS, saccades and blink frequency are computed internally. Data is sampled at $60 \mathrm{~Hz}$ and is interpolated off-line to fit the $10 \mathrm{~ms}$ step of the simulator logfile. Thanks to the accuracy of synchronization, excellent fit is obtained with the EOG-data.

Video recording. Using a video quad processor four individual video signals are merged to a single signal in PAL format consisting of four sub-screens. These show different views of the subject (camera view from the front and faceLAB view with detail on the eyes) as well as a view of the actual driving scene, and a selection of the instantaneous simulation parameters to directly identify the current car location and the driving parameters. The recorded signal is available in full resolution on digital video tapes and compressed .avi-files (MPEG4).

\section{Lane Departure Warning System}

An in-house developed LDWS warns the driver depending on his lane position, speed, heading angle and supposed lack of "intentional behavior" (braking, turning lights, etc.). It combines several advantages in comparison to the usual "hardware" rumble-strips solution allowing earlier 
and more flexible warning patterns, depending on the driving situation and the driver conditions (distraction or drowsiness). The software algorithm of the real assistance system is implemented into the driving scenario using the scenario parameters in place of the real vehicle sensors and

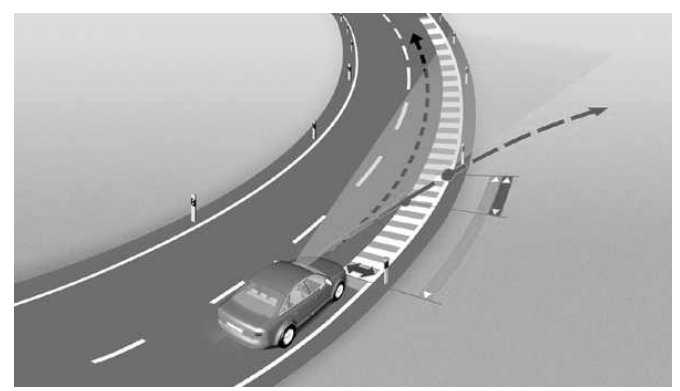
video signal for the lane border recognition (Figure 1). The two-level auditory warning signal depends on the reaction of the driver. When the algorithm foresees a LDE it emits a directional rumble strip noise. In case of an appropriate reaction within $400 \mathrm{~ms}$, the alarm stops, otherwise a second warning level is issued, shifting the noise towards the center of the road and ending with a bell tone (exp. details: Rossmeier, 2005).

Figure 1. Lane Departure Warning System, with lane border and trajectory recognition.

\section{EXPERIMENTS}

Simulator scenario. The driving scenario is divided into three different sections: (1) baseline - a simple 6-km segment with no fog, no curves, and almost no traffic; (2) control and test - a 9-km segment with sudden, large changes in curvature and slope with oncoming traffic, pedestrians as well as changing visibility; and (3) induction - a segment consisting of a six-fold exact repetition of an 18-km stretch in fog (50-m visibility, speed limit $50 \mathrm{~km} / \mathrm{h})$ with gentle curves and slopes, very low traffic density and only one marked local event (dangerous slope and curve).

The control and test sections precede and follow respectively the stimulus deprived drowsiness induction. The six-fold repetition of the induction track and the marked event included in each of them allow accurate comparison of the driver performance and its degradation during the trial. The complete driving task lasts about $2.5 \mathrm{hrs}$.

Cohort selection. To reduce inter-individual variability a homogeneous cohort of 63 healthy young men (22 to 27 yrs.) is chosen among 345 applicants, most of who were discarded because of liability to motion sickness. Second reason for rejection was wearing glasses because of possible interferences with the eye-tracking system. Subjects have to comply with the German traffic regulations, are neither informed about the objectives or the length of the trials nor had been exposed to a LDWS before. About one third (19) of the cohort drives were assisted by the LDWS and will later be referred to by the index 2 .

Trial protocol. A very detailed protocol ensures careful and consistent interaction with the subjects. The full trial lasts about 6 hours, starting at $10 \mathrm{am}$. The subject starts by filling out a questionnaire about his actual mood and feelings (MDBF, see Rossmeier, 2005) to determine a baseline of nervousness and drowsiness. Afterwards, he drives a short training scenario to get acquainted with the simulator and is invited to a rich meal with neither alcohol nor coffee. After application of the physiological sensors and a concentration test ( $\mathrm{d} 2$, see Rossmeier, 2005), the driving task starts at about $1: 30 \mathrm{pm}$ and lasts until approximately $4 \mathrm{pm}$. Control concentration test (d2), drowsiness questionnaire (MDBF) and, for the assisted subjects, a LDWS-evaluation questionnaire complete the protocol. 


\section{RESULTS AND OBSERVATIONS}

\section{Trials Statistics}

In post-processing, all data files (from the simulator, the physiology, the eye-tracking, etc.) are merged into a single data file, with synchronized step of $10 \mathrm{~ms}$. From the 63 trials, 53 are rated valid, 36 belonging to the non assisted sub-cohort (1), 17 using the LDWS (2). The mainly monotonous driving task results in a successful drowsiness induction with several hundred micro-sleep episodes detected by electrooculogram (EOG) and video signal (eye closure \% and PERCLOS) and confirmed by a double-blind behavioral analysis of video sequences by a trained team of independent observers (Kolrep, 2005). Both the physiology measurements (heart rate, electrodermal activity EDA, electroencephalogram EEG and electromyogram EMG) and the records of the driving behavior yield a coherent picture of these events. Figure 2 reports a microsleep event followed by a LDW so as to avoid a potential lane departure.

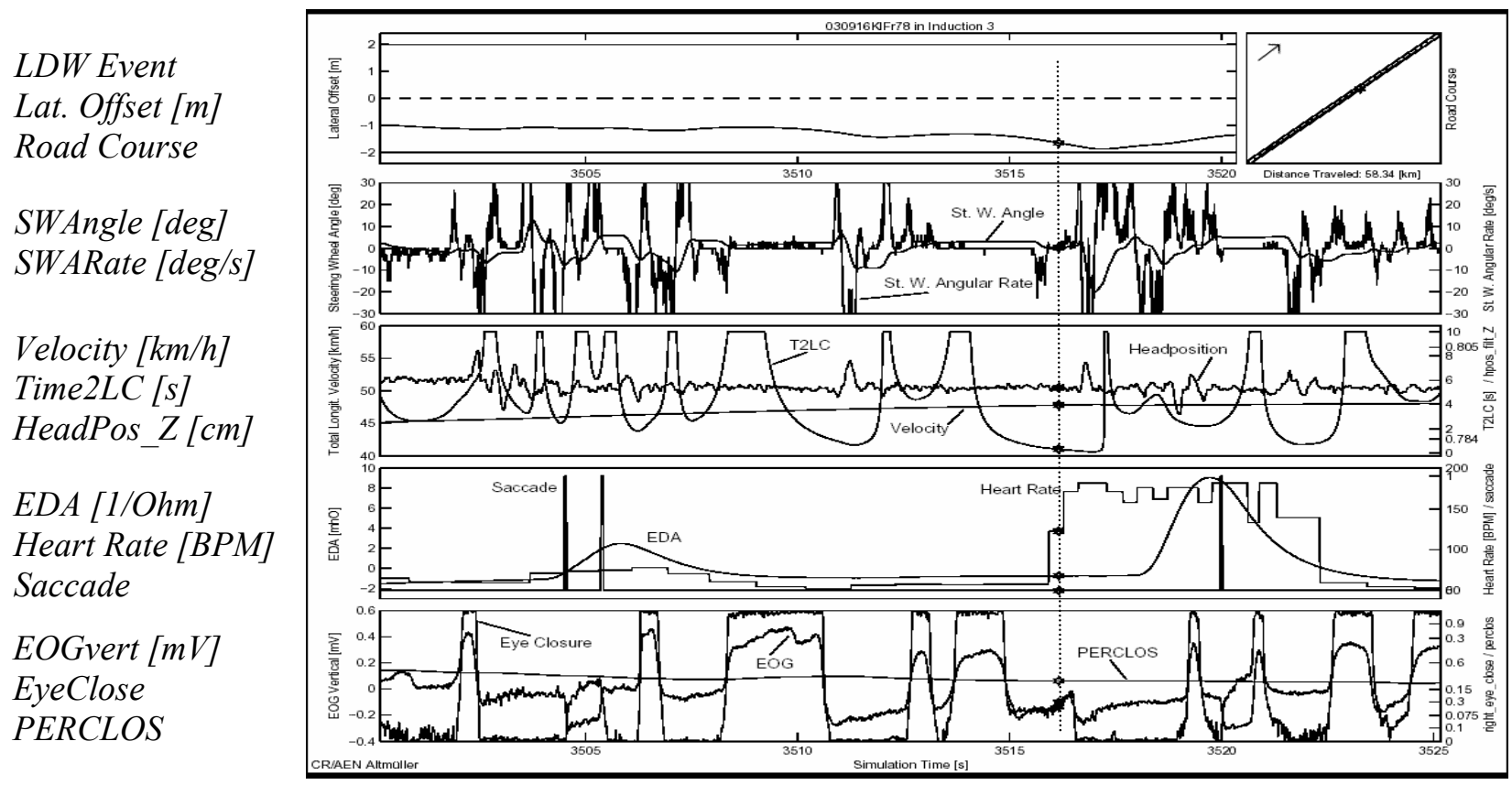

Figure 2. Micro-sleep episode and LDW (alarm time as dotted line). Lateral position, steering angle \& rate, velocity, time-to-line-crossing, head position, EDA, heart rate, saccade, vertical EOG, eye closure percentage and PERCLOS are represented over time (30 $\mathrm{s}$ interval)

\section{Lane Departure and Alarm Events}

Event description. During the driving task of the assisted sub-cohort 813 LDW occur, 784 of them caused by the 17 valid subjects. They are analyzed and sorted according to the causes and situations in which they arise and the different subjects' reactions. Following parameters are used among others to characterize the events:

- subject and alarm number, time, position, road curvature, road side;

- alarm cause and behavioral drowsiness rating; 
- eye closure, saccades and PERCLOS, electrodermal activity;

- reaction time and direction; and

- eventual LDE with associated departure magnitude, duration and out-of-lane-area.

LDW causes. Different rating methods are applied to determine the alarm cause: subjective rating of video material of the alarm event by two raters (RB) (Rossmeier, 2005), eye closure percentage within $20 \mathrm{~s}$ before the alarm (EC), drowsiness scale from $90 \mathrm{~s}$ video material

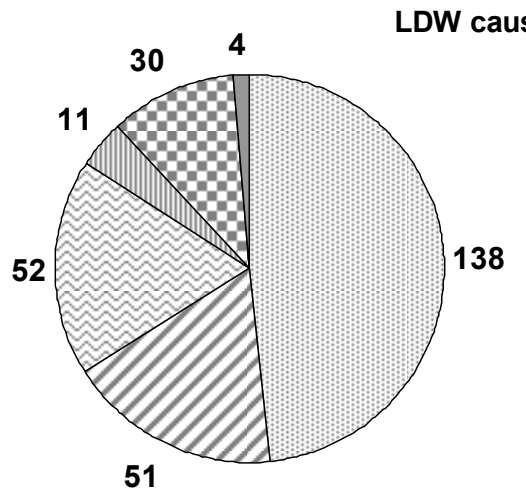

screening (HFC) including the alarm. Among the 784 LDW, 286 are caused by sleep as detected by at least RB or EC scales (Figure 3). The HFC analysis of 76 video sequences within this subset confirms that in 67 cases the subjects are very sleepy or showed micro-sleep, 8 are drowsy and only one is rated close to average. A highly significant relationship is found between the different scales $\left(\chi^{2}(1)=230.0, p<0.001\right)$.

Figure 3. Comparison of $\mathrm{RB}$ and EC rating for $\mathrm{LDW}$ causes of 286 alarm in drowsy situations

Subject distribution. The very uneven distribution of the alarm number per subject suggests a further subdivision of the sub-cohort 2: two distinct groups can be easily identified containing

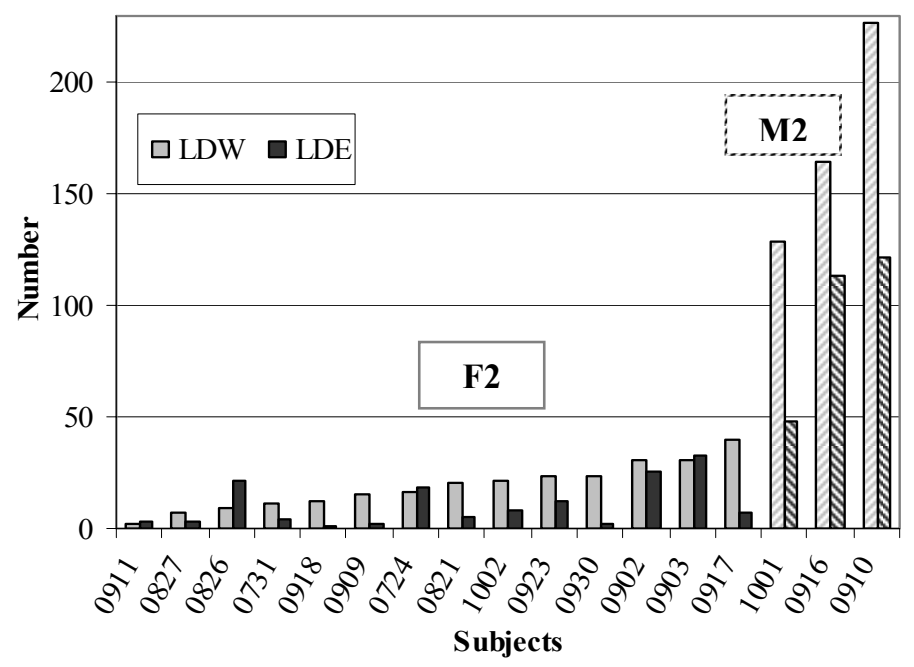
respectively 14 drivers with few alarms (F2) and 3 drivers with many alarms (M2) (Figure 4). The same applies to the number of LDE for both the assisted and the non-assisted subcohort. The latter splits into 29 drivers with few LDE (group F1) and 7 drivers with many LDE (group M1). Very different driving behavior describes the sub-groups: in spite of representing $80 \%$ of the subjects, the F1 and F2 drivers generate only $47 \%$ and $34 \%$ of the lane departure events in the own sub-cohort.

\section{Figure 4. Distribution of lane departure warnings and} events for the assisted groups F2 and M2

Lane Departure Events and Lane Departure Warnings. The F2 group with LDWS generates an average of only $10 \mathrm{LDE}$ per subject within the whole foggy induction, remaining approximately constant $(\mathrm{M}=1.7, \mathrm{SD}=0.16)$ over all six repetitions, in contrast with $20 \mathrm{LDE}$ per subject by the non-assisted group F1 with a highly significant increase over time $(\mathrm{T}(56)=3.48 ; \mathrm{p}=0.001)$. Moreover, the F2 events show less duration (-20\%), maximum departure (-36\%) and out-of-lane 
area $(-53 \%)$. The severity of the driving error increases for both the F1 and F2 groups in the last repetition, possibly showing habituation to such a warning system. Further analysis of the behavior in the foggy drowsiness induction segment shows that in group F2, only 25 of 145 LDE followed a warning and that, on the other hand, only (the same) $25 \mathrm{LDW}$ (out of 215) were followed by a lane departure (intersection in Figure 5). The excellent efficacy of the timing and warning design of the LDWS is shown in Table 1, with special regard to the reduction of the LDE caused by a micro-sleep episode ( $4^{\text {th }}$ column): only 6 LDE occur after 37 issued warnings.

Table 1. LDW and LDE in induction. For the assisted group F2 as in Figure 5: pale grey the total number of LDW; dark grey the total number of LDE; italics the intersection; bold the data of the sleepy events. For the non-assisted group F1: black the (much more severe) data.

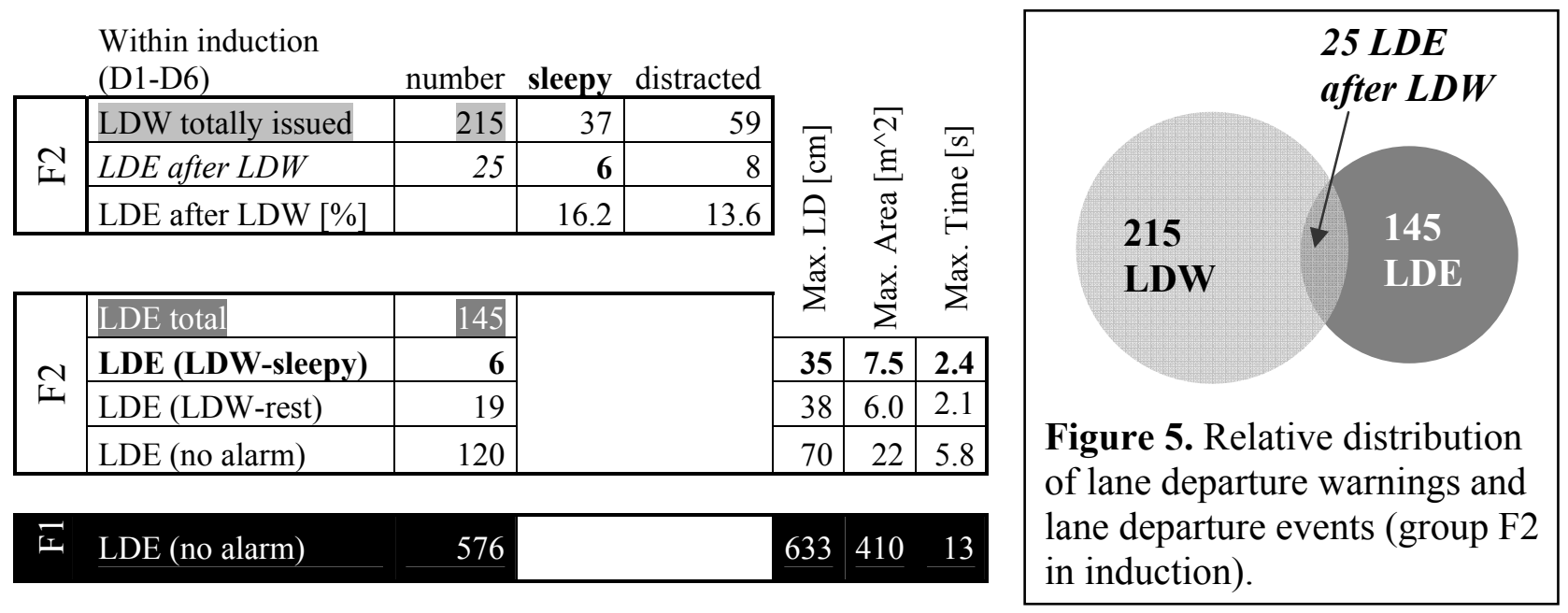

More detailed and specific investigation will also determine the causes of these 120 "missing" LDW and whether they can be ascribed exclusively to intentional behavior and therefore be "overlooked" in the perspective of not annoying the driver with unnecessary information.

Can group M2 be helped? This little set of only 3 subjects generates alone 520 LDW: 249 are caused by drowsiness (all but 1 in induction), 92 of which are followed by a LDE. The statistics

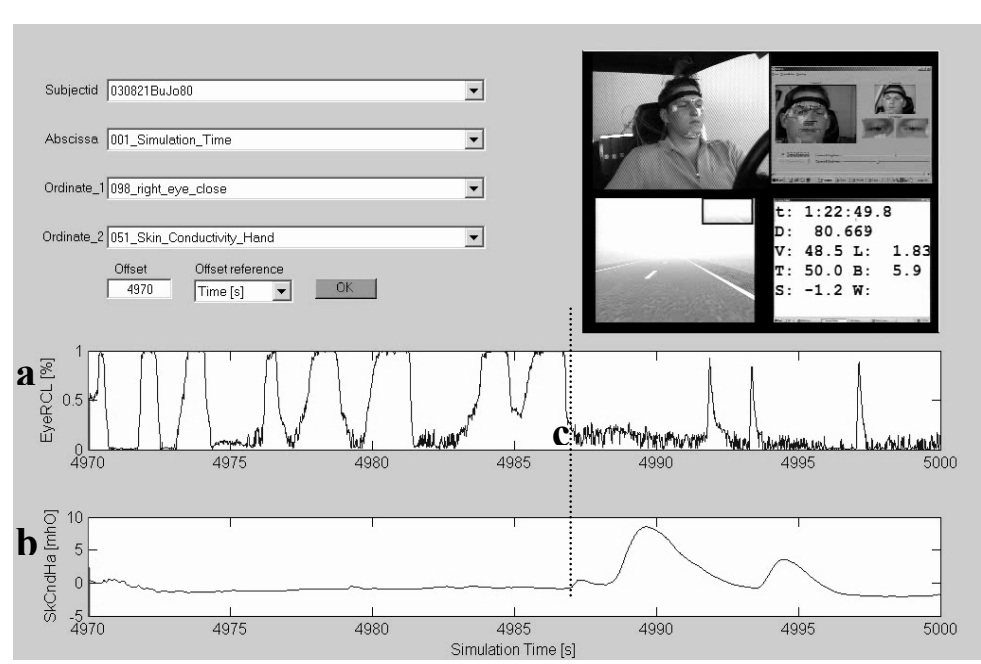
of the LDE is better than expected with $40 \mathrm{LDW}$ under $25 \mathrm{~cm}$, another 28 under $50 \mathrm{~cm}$ and only 14 severe errors over $1 \mathrm{~m}$. All the worst, some crossing the whole lane of the incoming traffic, occur in the last repeat. In contrast to Figure 6 where the subject responds to the alarm with a pronounced EDA peak and sharp blinks, the subjects sometimes show flat EDA curves and drowsy behavior. Here again a possible hint to habituation to the LDWS.

Figure 6. Synchronized selection of video and physiology (a: eye closure, b: EDA, c: alarm) 
The subjective assessment (MDBF) confirms a generalized increase of drowsiness in all subjects $(\mathrm{T}(52)=2.00, \mathrm{p}<0.001)$, while, at the same time, a highly significant $(\mathrm{T}(51)=2.00, \mathrm{p}<0.001)$ performance increase in the concentration test (d2) at the end of the driving task shows the availability of mental reserves. This experiment can therefore be described as pertaining to the time-on-task / deprivation category; further development of the LDWS to effectively contrast drowsiness will need to compare herewith test results obtained with sleep-deprived subjects.

\section{CONCLUSIONS}

The different perspectives on the Lane Departure Events and Lane Departure Warnings, as a result of physiological measurements and video analysis, yield a coherent picture of what happens before, during, and after a micro-sleep event. Because of a high number of micro-sleep episodes, the experiment design seems appropriate to measure effects of drowsiness on lane keeping behavior. We show that the LDWS strongly reduces the number and severity of the lane departure events even in case of a micro-sleep episode. System limits and the critical issue of habituation will be further addressed in future experiments.

\section{ACKNOWLEDGMENTS}

We thank Michael Dorna for the implementation of the lane departure algorithm, Dietrich Manstetten for the enduring theoretical and practical support, Yves Roland Nono Komguep for the accurate and patient characterization of the LDW.

\section{REFERENCES}

Allen, R.W., Rosenthal, T., Aponso, B.L., Klyde, D.H., Anderson, F.G., Chrstos, J.P. (1998). A low Cost PC Based Driving Simulator for Prototyping and Hardware-in-the-Loop Applications. SAE Paper No. 98-0222, Spec. Pub. 1361.

Altmueller, T., Manstetten, D., Rimini-Doering, M., Ladstaetter, U., \& Wolf, W. (2003). Evaluation of Driving Performance and Possible Criteria to design a Performance Feedback: a Driving Simulator Study. Proc. Intern. Conference on Vision in Vehicles, Granada, Spain

Altmueller, T. \& Wolf, W. (2003). Integration and Synchronization of Proprietary Measurement Components in a Driving Simulator Environment. Proc. Int. Conf. on Material Testing, Nuremberg AMA Service GmbH, p. 273-277

European Commission. (2001). Halving the number of road accident victims in the EU by 2010. Directorate General for Energy \& Transport, Brussels, Belgium

Kolrep, H., Rimini-Doering, M. et al. (2005). Wie sieht müde aus ? - Entwicklung und Validierung einer Skala zur Müdigkeitsbewertung von Kraftfahrern. Proc. 6. ZMMSWerkstatt VDI-Verlag, Reihe Nr. 22, Berlin, Germany

Langwieder, K. \& Sporer, A. (1994). Struktur der Unfälle mit Getöteten auf Autobahnen im Freistaat Bayern im Jahr 1991. HUK-Verband, München, Germany

Motoyama, S., Ohta, T., Watanabe T., Ito, Y. (2000). Development of Lane Departure Warning System. Proc. 7th ITS World Congress. No. 3270, Turin, Italy 
Nono Komguep, Y.R. (2004) Auswirkung eines Spurverlassenswarners auf Fahrverhalten und Fahrerzustand bei Müdigkeitsversuchen im Fahrsimulator. Diploma Thesis, FH Mannheim, Germany

Rimini-Doering, M., Manstetten, D., Altmueller, T., Ladstaetter, U., Mahler, M. (2001). Monitoring Driver Drowsiness and Stress in a Driving Simulator. Proc. Intern. Driving Symposium on Human Factors in Driver Assessment, Training and Vehicle Design Aspen, USA

Rossmeier, M., Grabsch, H.-P., Rimini-Doering, M. (2005). Blind flight: Do auditory lane departure warnings attract attention or actually guide action? Proceedings of ICAD 05 Eleventh Meeting of the International Conference on Auditory Display, Limerick, Ireland 